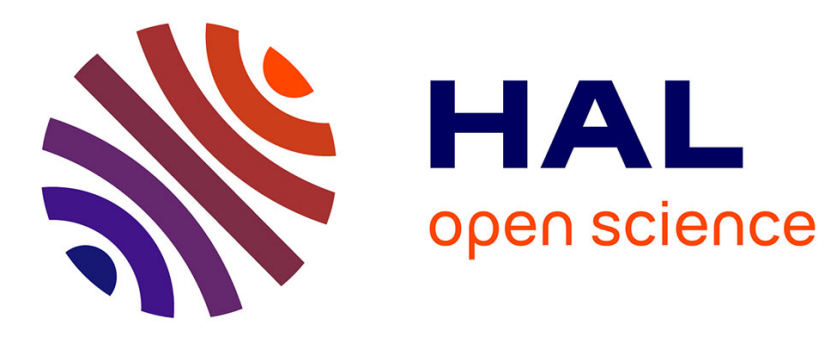

\title{
Dynamic node allocation in Network Virtualization
}

\author{
M. Said Seddiki, Ye-Qiong Song, Mounir Frikha
}

\section{To cite this version:}

M. Said Seddiki, Ye-Qiong Song, Mounir Frikha. Dynamic node allocation in Network Virtualization. HPCS - The 2013 International Conference on High Performance Computing \& Simulation - 2013, Jul 2013, Helsinki, Finland. 2013. hal-00877580

\section{HAL Id: hal-00877580 \\ https://hal.inria.fr/hal-00877580}

Submitted on 28 Oct 2013

HAL is a multi-disciplinary open access archive for the deposit and dissemination of scientific research documents, whether they are published or not. The documents may come from teaching and research institutions in France or abroad, or from public or private research centers.
L'archive ouverte pluridisciplinaire $\mathbf{H A L}$, est destinée au dépôt et à la diffusion de documents scientifiques de niveau recherche, publiés ou non, émanant des établissements d'enseignement et de recherche français ou étrangers, des laboratoires publics ou privés. 


\title{
Dynamic node allocation in Network Virtualization
}

\author{
M. Said Seddiki ${ }^{\dagger} *$, Ye-Qiong Song *, Mounir Frikha ${ }^{\dagger}$ \\ $\dagger$ Higher School of Communications of Tunis, University of Carthage, Tunisia \\ * LORIA Research Laboratory, University of Lorraine, France \\ $\dagger\{$ seddiki.said, m.frikha\} @ supcom.rnu.tn \\ * $\{$ ye-qiong.song $\} @$ loria.fr
}

\section{POSTER PAPER}

\begin{abstract}
Network virtualization is a new technology that provides a transparent abstraction of the networking resources. The most important challenge in network virtualization is the allocation of the physical substrate network among the pool of the active virtual networks (VNs). Our work in progress aims at allocating the node resources in a virtualized networking infrastructure. We believe that this allocation should be dynamic to lead to higher performance and better utilization of the physical resources. In this paper, we propose two models for dynamic node allocation for multiple VNs. The first model uses game theory and market-based approach in order to better allocate the physical node. The second one proposes a dynamic weighted round robin (WRR) approach where each $V N$ receives a fraction of the physical node according to an estimation of its current number of waiting packets and its weight. Both models use a distributed approach to minimize the packet delays inside the physical router and to fairly allocate the nodes between different $V N$.
\end{abstract}

Keywords-Network Virtualization; Resource allocation; Game theory; Queuing theory.

\section{INTRODUCTION}

In the recent years, network virtualization has been introduced to provide a solution for the ossification issue of the Internet [1]. This technology splits the role of Internet service provider ISP into two different roles [2]; the service provider (SP) and the infrastructure provider (InP). The InP is responsible for deploying and managing the physical network substrate. The service provider leases resources from multiple InPs and creates VNs by deploying customized protocols. Allocating physical resource for multiple SPs is a difficult task for the InP. The resource allocation approaches are classified into static and dynamic [3]. The dynamic approach offers a higher utilization by reallocating the resource to various VN instantiations. This work aims at maximizing the node throughput and to minimize the packet processing delay.

Game theory offers an efficient tool to model, analyze, and solve resource allocation problems involving multiple rational decision makers that strategically interact with each other in intelligent way. Queuing theory also provides an interesting mathematical tool to model and analyze the physical router

* This work has been partially supported by ANR Quasimodo (under No. ANR 2010 INTB 0206 01) where a packet needs to be processed by multiple pipe-lined resources from the input ports to the output ports.

In this paper, we study the resource allocation problem for multiple VNs with the goal of maximizing the number of VNs provisioned. We focus on how the VNs share a physical node in a fair and efficient way. We proposed two models using game theory and queuing theory to fairly allocate the node resources and to find out bottleneck nodes in order to prevent physical node downtime and performance degradation.

This paper is organized as follows. Section II describes the related work and the literature review. The two models for dynamic node allocation are proposed in section III. Results of the proposed models are presented in terms of performance in section IV. Section V concludes the paper and suggests the future work.

\section{RELATED WORK}

The most critical issue in Network virtualization is the Virtual Network Embedding (VNE) problem [4]. It is a three steps process that deals with the efficient mapping of the virtual network and the optimal constrained allocation of the shared physical resources in the substrate network [5]. The first step is the resource discovery which is usually performed by the InP to share the current state of the monitored physical network with multiple SPs. The second one is virtual network mapping which is performed by the SPs to match their requests with the available network resources [6]. The third one, which is the step addressed in this work, concerns the virtual network allocation. It is performed by the InP upon the reception of all the allocation requests from the SPs. Several research projects on network virtualization such as CABO [7] and 4WARD [8] addressed the VNE issue.

Many researchers have also extensively studied the resource allocation problem in network virtualization. The allocation is performed by $\mathrm{InP}$ once it receives a VN request. The InP should find the optimal resource allocation for both nodes and links in order to avoid deterioration of the Quality of service (QoS) levels for the active VNs. This problem has been addressed using mainly heuristic approaches because it is considered as an NP-hard problem [9]. 


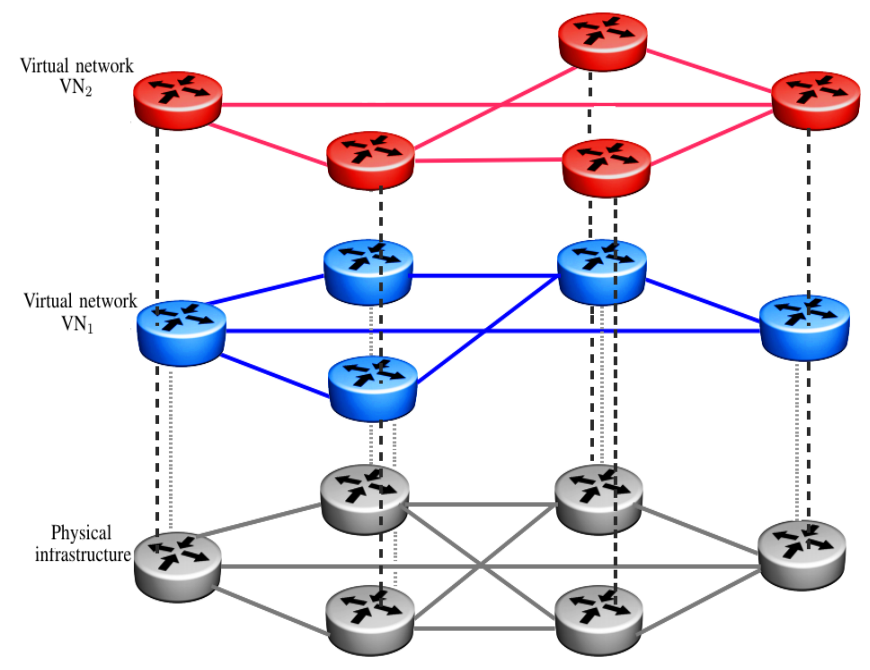

Figure 1. The virtualized networking environment

Figure 1 depicts an example of a virtualized networking environment where two $\mathrm{VN}$ are sharing the same underlying physical network. The VNs are deployed on top of a physical infrastructure that can be managed by one or more InPs. Each $\mathrm{VN}$ is a virtual topology of a set of virtual nodes connected together by a set of virtual links. A portion of the shared physical link and node is assigned to each VN.

Razzaq and Rathore [10] proposed a technique to use the physical resources in an efficient way. The authors suggested mapping the vertices of the $\mathrm{VN}$ as closely as possible in the substrate network. Then, all the virtual edges are assigned to the shortest paths that satisfy their demands. The proposed technique optimizes the resource utilization by accommodating more VNs than the approach of greedy node while it increases the $\mathrm{VN}$ embedding time.

Houidi et al. [11] proposed heuristic optimization algorithms to address the resource allocation where each $\mathrm{VN}$ request is divided among several InPs. The problem is solved as a mixed integer program in order to increase the acceptance ratio compared to existing heuristic solutions. The approach does not take into consideration the migration of a $\mathrm{VN}$ and offers a limited flexibility for handling the $\mathrm{VN}$ requests.

Botero et al. [12] proposed a heuristic algorithm to solve the VN embedding problem based on the optimization theory. The algorithm takes into account the CPU demanded by the hidden hops when a virtual link is mapped to fairly allocate the resources for multiple VNs. It also offers the possibility to accept VNs that do not ask for any demand. The goal of the proposed approach is to leave as much resource as possible in the substrate network resources while mapping the virtual nodes and links with explicit demands.

Butt et al. [13] proposed a mechanism for the selection among node and link resources based on their importance in the substrate network to prioritize some VNs over others. The mechanism also offers the re-optimization of the embedded $\mathrm{VN}$ requests. They concluded that the proposed mechanism improves the performance of the existing algorithms in terms of acceptance ratio and revenue-cost ratio.

He et al. [14] proposed a framework for Dynamically Adaptive Virtual Networks for a Customized Internet. The framework supports the periodic reassignment of bandwidth among multiple VNs. The proposed approach allows the use of multiple paths to reach a single node. Each path is selected to avoid the creation of bottlenecks. This approach can cause packetreordering problem.

In [15], the authors formulated a Markov random walk model to compute topology-aware resource ranking of nodes in a virtualized network environment. Based on the rank of each node, they proposed two new algorithms called RW-MaxMatch and RWBFS. The first one sorts virtual nodes in non-increasing order according to their rank values. The second algorithm constructs a breadth-first search tree where the root node is the virtual node with the largest rank value. The authors claimed that the proposed approach outperform the existing approaches in terms of the long-term average revenue and the acceptance ratio.

In this work, we address the problem of dynamic node allocation to satisfy the resource requirements of each $\mathrm{VN}$ while optimizing the use of the CPU cycles, the memory access, and the size of the buffer in the substrate node.

\section{DYNAMIC NODE ALLOCATION MODELS}

In this section, we develop two models for dynamic node allocation in a virtualized network infrastructure using game theory and queuing theory. We model the substrate network as a undirected graph and denote it by $G^{p}=\left(N^{p}, L^{p}\right)$ where $N^{P}$ is the set of substrate nodes and $L^{p}$ is the set of substrate links. The substrate node is associated with the CPU and memory capacity. The substrate link $l^{p}(i, j) \in \mathrm{L}^{p}$ between two substrate nodes $i$ and $j$ is associated with the bandwidth capacity. Each $\mathrm{VN}$, using the shortest algorithm, k-shortest paths, or multicommodity flow algorithms [16] performs the VN mapping. Once the mapping is done, each VN has to compete with other VNs in order to receive a fraction of each physical node.

We consider there are $n$ virtual networks, each with a budget, that are competing on $m$ physical nodes deployed and managed by multiple infrastructure providers. Through the proposed models, we aim at providing a fair allocation scheme between isolated concurrent VNs while they are sharing the same physical node.

\section{A. Dynamic node allocation modeling through game theory}

The first dynamic node allocation model is based on proportional share mechanism where each $\mathrm{VN}$ divides its budget among the multiple nodes and receives a fraction of each node proportional to its bid. We propose an approach similar to the work proposed by Feldman et al. [17] that was presented for allocating shared computational resources in computer networks. However, we extend the game using a prediction of the next bids in order to maximize the utilization of the physical node resources. The delay decreases when the utility 
function increases. The objectives of the proposed game are to maximize the VN's utility rather than focusing on the uniqueness of the Nash equilibrium.

We assign to each VN a fixed budget $G_{i}$. This budget depends on requested QoS in order to satisfy its end users. The higher is the requested QoS, the greater is the budget for the VN.

For a given $\mathrm{VN}_{i}$ the strategy in the game is to submit a bidding vector $\left(B_{i, j}\right)_{j=1, \ldots, m}$ for different physical node and then receives a fraction of each node that depends on its bid. The VN also assigns a weight for the requested physical node. The weights reflect the preference of the VN to each physical node. The total bids for $\mathrm{VN}_{i}$ are less than or equal to its initial budget $G_{i}$. We define the price $P_{j}$ of a node $j$ as the sum of all the bids submitted by all the active VNs for that node.

Thus, the fraction of the physical node $j$ received by a virtual network $\mathrm{VN}_{i}$ is given by:

$$
F_{i, j}=\frac{B_{i, j}}{P_{j}}
$$

The utility function is represented as a function of the fraction $F_{i, 1}, F_{i, 2}, \ldots F_{i, m}$, which it receives from each physical node and a vector of weight $w_{i, 1}, w_{i, 2}, \ldots w_{i, m}$ assigned to each node. It defines the player's preferences on the physical routers. Thus the utility function is a linear function defined as:

$$
U_{i}\left(F_{i, 1}, \ldots, F_{i, m}\right)=w_{i, 1} F_{i, 1}+\ldots+w_{i, m} F_{i, m}
$$

By maximizing its utility function, every $\mathrm{VN}$ decreases the delay for a packet to be processed by the physical nodes. In our reasoning, we assume that the $\mathrm{VN}$ is selfish trying to maximize its utility function and enhancing its performance by allocating the maximum of the node resource. In order to reach the Nash equilibrium state of the best response of $\mathrm{VN}_{i}$ is a solution of the following optimization problem:

$$
\left\{\begin{array}{l}
\text { Maximize } U_{i}=\left(\frac{B_{i, 1}}{P_{1}}, \frac{B_{i, 2}}{P_{2}}, \ldots \frac{B_{i, m}}{P_{m}}\right) \\
\text { Subject to } \quad \sum_{j=1}^{l} B_{i, j}=G_{i} \text { and } B_{i, j} \geq 0
\end{array}\right.
$$

In our approach, we assume that a $\mathrm{VN}$ will try to maximize its utility function by predicting the next bids. Each VN uses the past performance of the resource and its price in order to find the best response in the game and maximize its utility function. The key of the prediction is to find the bottlenecked physical node, which is the physical node where a packet for a given VN spends the maximum delay to be processed. Each VN has a bottlenecked resource at a given time. The main idea of the prediction is to minimize the packet delay in the bottlenecked resource and keep the same performance of the other resources. At each time of the game, the bottlenecked physical node changes. The next bid at $T+1$ instant for the $\mathrm{VN}_{i}$ when the physical node $j^{\prime}$ is considered as bottlenecked is equal to:

$$
B_{i, j^{\prime}}^{T+1}=P_{j^{\prime}}^{T} * F_{i, j^{\prime}}^{r e q}
$$

At $T+1$ instant, the virtual network $\mathrm{VN}_{i}$ tries to maximize his bid $B_{i, j}^{T}$ for the rest of non-bottlenecked nodes as a solution of the following optimization problem:

$$
\left\{\begin{array}{l}
\left(B_{i, j}^{T+1}\right)_{j \neq j^{\prime}}=\operatorname{argmax}\left(\left(B_{i, j}^{T}\right)_{j \neq j^{\prime}}\right) \\
\text { Subject to } \sum_{j=1}^{m} B_{i, j} \leq G_{i} \text { and } B_{i, j} \geq 0
\end{array}\right.
$$

\section{B. Dynamic node allocation using queuing theory}

This work aims at developing multiple schedulers for multiple physical nodes based on WRR algorithm. This scheduler is able to predict the requirement for different flows of packets for a given $\mathrm{VN}$ that needs to be processed by different physical nodes. Each VN is served on the basis of the weight assigned to the related queue. We aim at providing a higher isolation for different instance of VNs by allocating the physical node in a fair, adaptive and efficient way.

We suppose that a virtual network $\mathrm{VN}_{i}$ has a fixed global weight $G w_{i}$ that depends on the QoS requested to achieve its performance. We also suppose that the processing capacity of a single node $N_{j}$ with a WRR scheduler is $C_{j}$. If all the concurrent VNs are sharing this node, then each of them receives a fraction of that capacity. The minimum guaranteed service rate given by assigned weight for each $\mathrm{VN}$ is equal to:

$$
C_{i, j}^{T}=\frac{G w_{i, j}^{T} * C_{j}}{\sum_{k=1}^{N} G w_{k, j}^{T}}
$$

We use the exponential moving average to estimate the current input rate for each VN in each physical node at $T$ instant. The estimation of the input rate is given by the following equation:

$$
\bar{\lambda}_{i, j}^{T}=(1-\alpha) * \bar{\lambda}_{i, j}^{T-1}+\alpha * \lambda_{i, j}^{T}
$$

The packets arrive to the first physical node $N_{1}$ according to a Poisson process with a rate $\lambda$ equal to the sum of all the input rate of all the active VNs. The first physical node $N_{1}$ is considered as a queuing system with multiple queues and a single sever. The service rate is deterministic and the interarrival is exponential. In order to update the weights of each virtual network $\mathrm{VN}_{i}$ in the resource $R_{1}$, the dynamic WRR algorithm uses the estimation about the average number of packet in each queue at $\mathrm{T}$ instant according the estimation of the input packet arrival.

Let $A_{i, j}^{T}$ be the average number of waiting packets for a given virtual network $\mathrm{VN}_{i}$ at $T$ instant in order to be processed by the node $N_{1}$. According to Pollaczek-Khinchin formula [18] it is defined as:

$$
A_{i, 1}^{T}=\frac{\left(\bar{\lambda}_{i, 1}^{T}\right)^{2}}{2 C_{i, 1}^{T} *\left(C_{i, 1}^{T}-\bar{\lambda}_{i, 1}^{T}\right)}
$$

We define $D_{i, 1}^{T}$ as the average delay for packet for a given virtual network $\mathrm{VN}_{i}$ spent in the physical node $N_{1}$ at $T$ instant. This delay is equal to the average waiting delay plus 
the average service delay of the node. The average packet delay is given by the following equation:

$$
D_{i, 1}^{T}=\frac{1-\left(\bar{\lambda}_{i, 1}^{T} / 2 * C_{i, 1}^{T}\right)}{C_{i, 1}^{T}-\bar{\lambda}_{i, 1}^{T}}
$$

For the first physical node $N_{1}$, the new weight of a virtual network $\mathrm{VN}_{i}$ at $T+1$ depends of its global weight $G w_{i}$ and the estimation of its average number of waiting packet at $\mathrm{T}$ instant. At $T+1$ instant each virtual network $\mathrm{VN}_{i}$ receives a new weight in the node $N_{1}$ equal to :

$$
w_{i, 1}^{T+1}=\left(\frac{G w_{i}}{\sum_{k=1}^{N} G w_{k}}+\frac{A_{i, 1}^{T}}{\sum_{k=1}^{N} A_{k, 1}^{T}}\right) \frac{C_{1}}{2}
$$

For the other physical nodes $N_{j \neq 1}$, each virtual network $\mathrm{VN}_{i}$ receives a new weight according to its global weight $G w_{i}$ and the number of packets that have been already processed by the previous node $N_{j-1}$. Thus, at $T+1$ instant every virtual network $\mathrm{VN}_{i}$ receives a new weight equals to :

$$
w_{i, j \neq 1}^{T+1}=\left(\frac{G w_{i}}{\sum_{k=1}^{N} G w_{k}}+\frac{C_{i, j-1}^{T}}{\sum_{k=1}^{N} C_{k, j-1}^{T}}\right) \frac{C_{j \neq 1}}{2}
$$

\section{EXPERIMENTAL RESULTS}

In this section, we implement the proposed node allocation models using Matlab and present the experimental results. In order to discuss the performance results of the node allocation, we suppose that $3 \mathrm{VNs}$ are sharing 3 physical nodes for a given infrastructure provider. We try to simulate the fraction received for each $\mathrm{VN}$ during a time period. The virtual networks $\left(\mathrm{VN}_{1}\right.$, $\left.\mathrm{VN}_{2}, \mathrm{VN}_{3}\right)$ are competing for the nodes $\left(N_{1}, N_{2}, N_{3}\right)$. We assign three budgets $\left(G_{1}, G_{2}, G_{3}\right)$ for the VNs. We assume that $G_{1} \geq G_{2} \geq G_{3}$. The budget assigned for the VN depends on the level of the quality of service requested. All the VNs have a Poisson arrivals at rate $\lambda_{1}, \lambda_{2}, \lambda_{3}$ with fixed length packets. We implement the delays for processing a packet in each node. We suppose there is a random input packet flow for each physical node. We run our algorithm and we observe the packet delays in every node and how much fraction received by the VNs at $T$ and $T+1$ instants. Each VN predicts the next bids in order to find the best response during the game at $T+1$ instant.

Figure 2 illustrates the fraction received by the three concurrent VNs in order to get a fraction of the node $N_{1}$. Figure 3 shows the delay for packets of the virtual network of $\mathrm{VN}_{1}$ inside the node $N_{1}$.

According to both figures, the physical node $N_{1}$ was considered as a bottlenecked node for the Virtual network $\mathrm{VN}_{1}$ between $\mathrm{T}=22 \mathrm{~ms}$ and $\mathrm{T}=25 \mathrm{~ms}$. Thus, $\mathrm{VN}_{1}$ increased its bid in order to receive a higher fraction and decrease the packet delay inside the node $N_{1}$.

For the second model of node allocation, we assign three global weights $\left(G w_{1}, G w_{2}, G w_{3}\right)$ for the three virtual networks. We assume that $G w_{1}>G w_{2}>G w_{3}$, and the

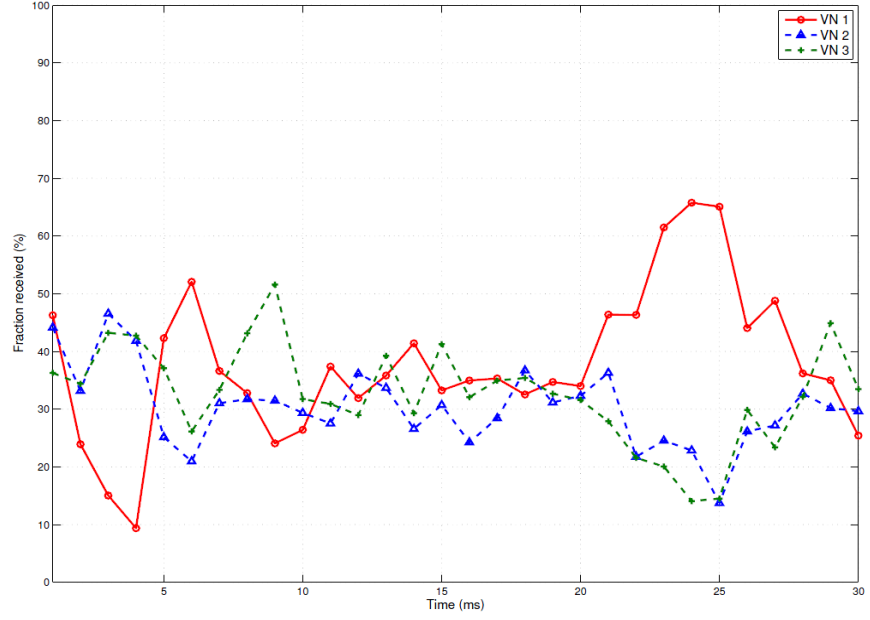

Figure 2. The fractions of the node $N_{1}$ received by the three concurent VNs during a given time interval

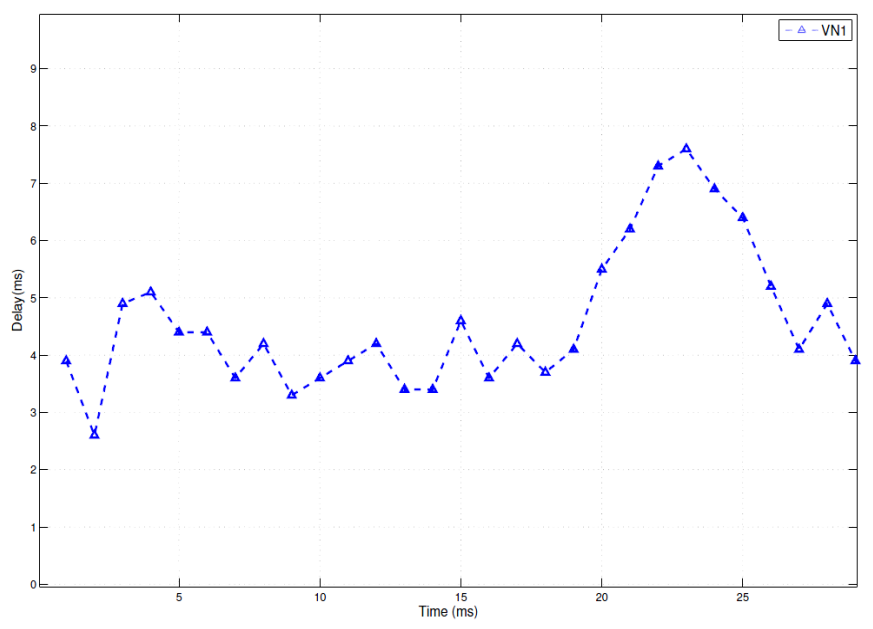

Figure 3. The packet delay inside the physical node $N_{1}$ for packets of the virtual network $\mathrm{VN}_{1}$

weights assigned to the $\mathrm{VN}$ depend on the level of the requested QoS where $G w_{1}=50, G w_{2}=30$, and $G w_{3}=20$. The queuing system is supposed to be stable such as the sum of the arrival rates of all the VNs is always less than the node capacity. We update dynamically the weight of each $\mathrm{VN}_{i}$ and we compare the results obtained by the static and dynamic weighted round robin when the arrival rate is the same and the exponential moving average parameter $\alpha$ is equal to 0,5 . The performances of our simulation are expressed in terms of packet delays, the number of waiting packets in different queues for different VNs and the efficient usage of the physical nodes.

Figure 4 shows the fraction received by the virtual network $\mathrm{VN}_{1}$ of the physical node $N_{1}$, that has a processing capacity of 2000 packets/s, with the proposed dynamic WRR algorithm and the static one. Figure 5 illustrates the average packet delay 


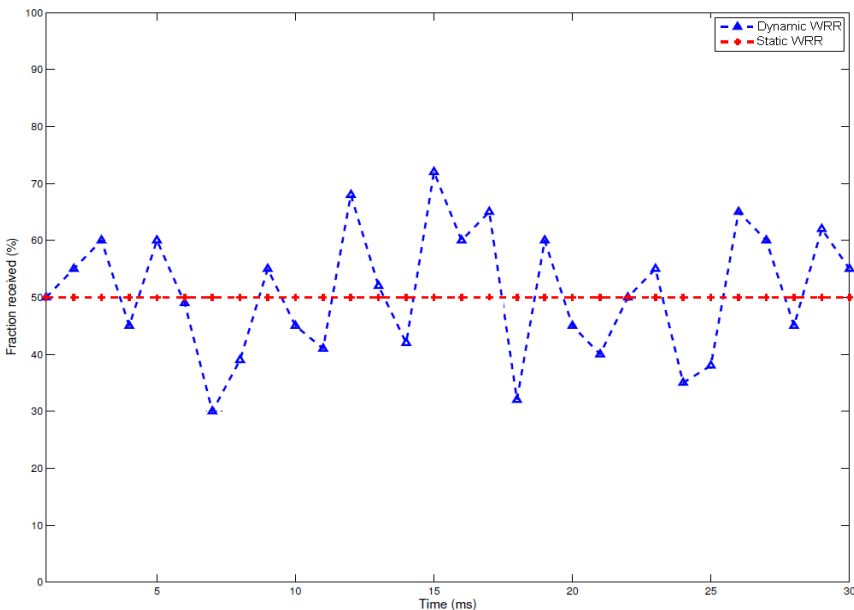

Figure 4. The fractions of the node $N_{1}$ received by the virtual network $\mathrm{VN}_{1}$

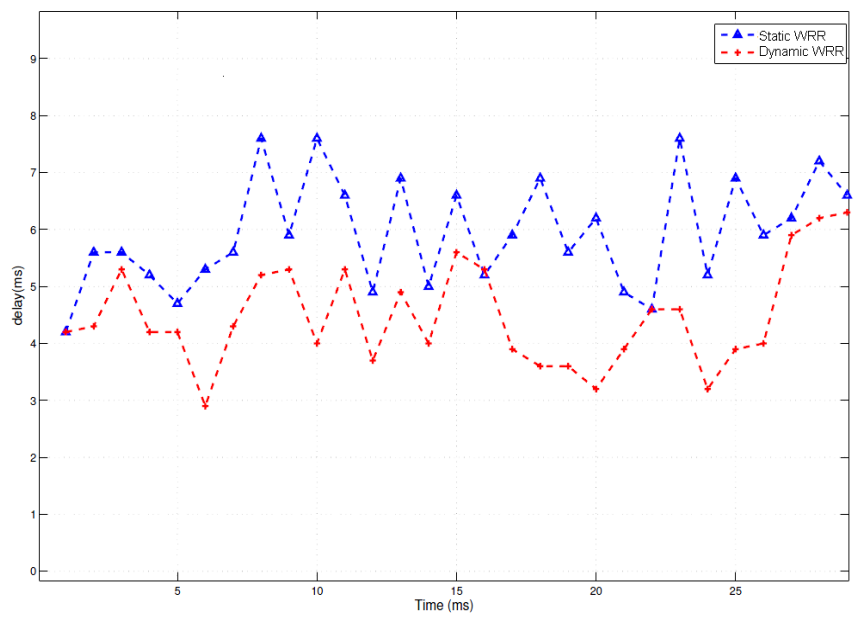

Figure 5. The average delay for the packets of $\mathrm{VN}_{1}$ inside the node $N_{1}$

of the packets of the virtual network $\mathrm{VN}_{1}$ inside the physical node $N_{1}$. We can clearly conclude that our approach achieves better results than the static approach in terms of minimizing the packet delay inside a physical node.

\section{CONCLUSION}

In this paper, we applied game theory and queuing theory concepts to solve the problem that occurs where a physical node is allocated dynamically to a set of VNs. Our main contribution is to use a prediction of the current workload in order to share the node among multiple instances of VNs and avoid service degradation. We have shown that both models offer higher utilization of physical node and better managing the satisfaction of virtual networks by minimizing the packet delays inside the physical node.
The future steps consist in exploring other distributions of packet inter-arrival rates at each node that could be more realistic for Internet-like traffic [19]. We will also propose an admission control for each model in order to bind the limit for creation requests for a virtual network and to prevent it from deteriorating the performance of the active VNs.

\section{REFERENCES}

[1] N. M. M. K. Chowdhury and R. Boutaba, A Survey of Network Virtualization, Computer Networks, vol. 54 (5), 862-876 (2010).

[2] Chowdhury N. M. M. K. and Boutaba R., Network virtualization: State of the art and research challenges, IEEE Communications Magazine, vol. 47 (7), 2026 (2009).

[3] A. Haider, R. Potter, A. Nakao, "Challenges in Resource Allocation in Network Virtualization", Proceedings of 20th ITC Specialist Seminar, Hoi An, Vietnam, 2009.

[4] A. Fischer, J. F. Botero, M. Beck, H. De Meer, and X. Hesselbach, "Virtual Network Embedding: A Survey," IEEE Communications Surveys and Tutorials, vol.PP, no.99, pp.1,19.

[5] M. A. Wang, R. Dutta, G. N. Rouskas, and I. Baldine, "Network Virtualization: Technologies, Perspectives, and Frontiers," Journal ofLightwave Technology, vol.31, no.4, pp.523,537, 2013.

[6] W. Hsu, Y. Shieh, "Virtual network mapping algorithm in the cloud infrastructure", Journal of Network and Computer Applications, 2013.

[7] N. Feamster, L. Gao, and J. Rexford, "How to lease the internet in your spare time", SIGCOMM Computer Communication Review, vol. 37, no. 1, pp. 6164, 2007.

[8] N. Niebert, S. Baucke, I. El-Khayat, M. Johnsson, B. Ohlman, H. Abramowicz, K. Wuenstel, H. Woesner, J. Quittek, and L. Correia, The way 4ward to the creation of a future internet, in Proc. of the 19th IEEE International Symposium on Personal, Indoor and Mobile Radio Communications (PIMRC08), pp. 15, 2008.

[9] A. Belbekkouche, M. M. Hasan, A. Karmouch, Resource Discovery and Allocation in Network Virtualization, IEEE Communications Surveys and Tutorials, Volume: PP, Issue 99, pp. 1-15, 2012

[10] A. Razzaq and M. S. Rathore, An approach towards resource ef?cient virtual network embedding, in Proc. International Conference on High Performance Switching and Routing (HPSR10), 2010.

[11] I. Houidi, W. Louati, and D. Zeghlache, A distributed virtual network mapping algorithm, in Proc. IEEE International Conference on Communications (ICC08), 2008.

[12] J. F. Botero, X. Hesselbach, A. Fischer, and H. de Meer, Optimal mapping of virtual networks with hidden hops, Telecommunication Systems, vol. 52, 2013.

[13] N. F. Butt, N. Chowdhury, and R. Boutaba, Topology-awareness and re-optimization mechanism for virtual network embedding, in Proc. 9th IFIP NETWORKING Conference, 2010.

[14] J. He, R. Zhang-Shen, Y. Li, C. Lee, J. Rexford, and M. Chiang. Davinci: Dynamically Adaptive Virtual Networks for a Customized Internet, in Proc of ACM CoNEXT, 2008.

[15] X. Cheng, X. Su, Z. Zhang, H. Wang, F. Yang, Y. Luo, and J. Wang, "Virtual network embedding through topology-aware node ranking", Computer Communication Review, Vol. 41, no. 2, pp- 38-47, 2011.

[16] N. Chowdhury, M. Rahman, and R. Boutaba, "Virtual network embedding with coordinated node and link mapping," in Proc. of INFOCOM 2009, IEEE, pp. 783791, 2009.

[17] M. Feldman, K. Lai, and L. Zhang, Allocation Market for Computational Resources, IEEE Transaction Parallel Distributed Systems, Proceedings of 20th ITC Specialist Seminar, pp.1075-1088, 2009.

[18] P. Pochec and W. Mardini, Modelling with queues: an empirical study, in Proc. of the IEEE Canadian Conference on Electrical and Computer Engineering, pp 685-689, 2001.

[19] A. Adas, "Traffic models in broadband networks", IEEE Communications Magazine, vol.35, no.7, 1997, pp.82-89. 